\title{
Neue Herausforderungen für den Datenschutz
}

\section{3./04. November 2010}

Zum Abschluss der 80. Konferenz der Datenschutzbeauftragten des Bundes und der Länder hat der diesjährige Konferenzvorsitzende, der baden-württembergische Datenschutzbeauftragte Jörg Klingbeil, gemeinsam mit dem Bundesbeauftragten für den Datenschutz und die Informationsfreiheit, Peter Schaar, und dem Bayerischen Landesbeauftragten für den $\mathrm{Da}$ tenschutz, Dr. Thomas Petri, die Konferenzergebnisse vorgestellt. Bayern wird die Konferenz 2011 ausrichten.

Die Konferenz der Datenschutzbeauftragten des Bundes und der Länder beobachtet mit Sorge die Tendenz, dass die Informationssysteme der Sicherheitsbehörden zunehmend in Volltextsysteme umgewandelt werden. Dies hat zur Folge, dass auch die bisherigen Akteninhalte elektronisch recherchierbar den Sicherheitsbehörden zur Verfügung stehen, unabhängig von welcher Stelle die Daten eingestellt wurden. Somit kann aus jedem Vorgang jede dort erwähnte Person, völlig unabhängig davon, ob sie für die Sicherheitsbehörden relevant ist oder nicht, herausgesucht werden. Dieser Paradigmenwechsel steht im Widerspruch zum geltenden Recht. Die bisherigen datenschutzrechtlichen Sicherungen würden aufgehoben. Die verfassungsrechtlich gebotenen Vorkehrungen zum Schutz des Rechts auf informationelle Selbstbestimmung werden hinfällig, wenn die elektronische Volltexterfassung vieler Informationen der Sicherheitsbehörden zugelassen würde. „Google darf nicht das Vorbild für die Recherche in den Dateien von Polizei und Verfassungsschutz sein“, erklärte der Vorsitzende der Konferenz, Jörg Klingbeil, (siehe Dokumentation in diesem $\mathrm{Heft}^{1}$ ).

Seit Anfang 2010 sind Neubauten und renovierte Gebäude mit so genannten

\footnotetext{
1 Seite 48 in diesem Heft
}

Smart Metern auszurüsten, die den aktuellen Energieverbrauch im Sekundentakt aufzeichnen können. Solche digitalen Messgeräte erfassen sensible Verbrauchswerte aus Wohnungen wie Strom und Gas und gewähren dadurch tiefen Einblick in die Lebensgewohnheiten der Betroffenen. Dies kann nach Ansicht der Datenschutzbeauftragten das Grundrecht auf informationelle Selbstbestimmung und die grundrechtlich gesicherte Unverletzlichkeit der Wohnung beeinträchtigen. Die zur Einführung digitaler Zähler bisher erlassenen Rechtsnormen im Energiewirtschaftsgesetz schützen die Privatsphäre der Betroffenen allerdings nur unzureichend. Nach Ansicht der Datenschutzbeauftragten ist beim Betrieb effizienter Versorgungsnetze den Persönlichkeitsrechten absolute Priorität einzuräumen. „Wir brauchen gesetzliche Regelungen für die Erhebung, Verarbeitung und Nutzung der durch digitale Zähler erhobenen Verbrauchsinformationen“, so Jörg Klingbeil. „Dieser Bereich darf nicht unreguliert bleiben, denn das hieße, die schutzwürdigen Belange der Bürger den kommerziellen Interessen der Energieversorgungsunternehmen unterzuordnen. Die Datensouveränität der Bürger sollte oberste Priorität haben" (Siehe Dokumentation in diesem $\mathrm{Heft}^{2}$ ).

Die Bundesregierung will den Datenschutz mit Hilfe einer Stiftung Datenschutz stärken. Sie soll u.a. Produkte und Dienstleistungen auf Datenschutzfreundlichkeit prüfen und die Bildung im Bereich des Datenschutzes fördern. Die $\mathrm{Da}$ tenschutzbeauftragten des Bundes und der Länder unterstützen dieses Ziel ungeachtet noch offener verfassungsrechtlicher Fragen. Das Vorhaben setzt allerdings voraus, dass die Stiftung ihre Aufgaben unabhängig von den Daten verar-

\footnotetext{
2 Seite 48 in diesem Heft
}

beitenden Stellen und der IT-Wirtschaft wahrnimmt. Überdies kann die Stiftung nur solche Aufgaben übernehmen, die nicht ausschließlich den Datenschutzbeauftragten zugewiesen sind. Die Datenschutzbeauftragten bieten der Bundesregierung ihre Unterstützung an und erwarten, frühzeitig in die Überlegungen zu Stellung und Aufgaben der Stiftung einbezogen zu werden (siehe Dokumentation in diesem $\mathrm{Heft}^{3}$ ).

Ein weiteres wichtiges Thema bei der Datenschutzkonferenz war erneut die Vorratsdatenspeicherung. Hierzu hatte die Konferenz bei ihrer Frühjahrssitzung vor dem Hintergrund der Entscheidung des Bundesverfassungsgerichts vom 2. März 2010 ihre grundsätzlich ablehnende Haltung betont. Hieran hält die Konferenz fest. Angesichts der Forderungen von Sicherheitspolitikern nach einer schnellen Neuregelung der Vorratsdatenspeicherung halten es die Datenschutzbeauftragten für erforderlich, sich ernsthaft mit Alternativen zum massenhaften anlass- und verdachtslosen Speichern von Verkehrsdaten der Telekommunikation auseinanderzusetzen.

Schließlich befasste sich die Konferenz aus aktuellem Anlass auch mit der neuen Datenschutzstrategie der EU-Kommission und der Entwicklung des Datenschutzes auf internationaler Ebene.

Weitere Beratungspunkte waren die anstehende Volkszählung, der Zensus 2011, der seit wenigen Tagen eingeführte elektronische Personalausweis und der Beschäftigtendatenschutz. Der Bundesrat hat sich am 5. November 2010 mit dem Gesetzentwurf der Bundesregierung hierzu befasst. Die Datenschutzkonferenz wird den weiteren Fortgang des Gesetzgebungsverfahrens kritisch begleiten.

3 Seite 49 in diesem Heft 